\title{
Historia natural de retinopatía diabética en un estudio a largo plazo en pacientes con diabetes tipo 1 . Factores de riesgo para progresión a enfermedad proliferante
}

\author{
Juan Verdaguer ${ }^{1}$, Mario Zanolli ${ }^{1}$, G onzalo Sepúlveda ${ }^{1}$, \\ Manuel García de los Ríos², Angélica Domínguez ${ }^{3 a}$. \\ Natural history of diabetic retinopathy \\ in a retrospective cohort \\ of type 1 diabetics
}

Background: Diabetic retinopathy is one of the most common causes of blindness among adults. Aim: To report the natural history of diabetic retinopathy among Chilean patients with type 1 diabetes followed for a mean of 18 years. Material and methods: Retrospective review of medical records of 39 patients aged 26 to 70 years, (20 females, 78 eyes) with type 1 diabetes controlled by the same ophthalmologist from 1971 to 2008. A questionnaire was sent to each patient and their treating physician to request information about the evolution of the disease and metabolic control. Results: The questionnaire was answered by 24 patients (62\%) and 21 attending physicians (54\%). Small hard drusen were observed in 25 patients (64\%). In 12 cases the drusen were detected before the development of any type of retinopathy. Eleven women became pregnant and retinopathy progressed in four of them. Twently three patients (59\%) developed proliferative diabetic retinopathy (PDR). Patients with PDR had a significantly longer duration of diabetes and worse glycemic control. There was a higher frequency of diabetic nephropathy in the PDR group, but only 13 patients out of 23 with PDR had nephropathy. The retinopathy progressed to high risk PDR two years after successful kidney-pancreas transplantation in one patient. Conclusions. In patients with type 1 diabetes mellitus, small hard drusen may be the initial manifestation of diabetic retinopathy. Risk factors for progression to PDR were duration of diabetic and poor glycemic control. Nephropathy was more prevalent in patients with PDR, but a significant group of PDR patients did not have demonstrable nephropathy (Rev Méd Chile 2009; 137: 1145-52).

(Key w ords: Diabetes mellitus, type 1; Diabetic nephropathies; Diabetic retinopathy)

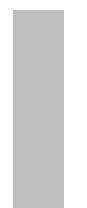

\footnotetext{
Recibido el 10 de noviembre, 2008. Aceptado el 5 de agosto, 2009.

Los autores no declaran apoyo financiero.

${ }^{1}$ Fundación Oftalmológica Los Andes, Universidad de los Andes. ${ }^{2}$ Departamento de Diabetes y Nutrición, Hospital San Juan de Dios, Universidad de Chile. ${ }^{3}$ Departamento de Salud Pública, Pontificia Universidad Católica de Chile, Santiago de Chile.

${ }^{a}$ Estadístico
}

Correspondencia a: Dr. Juan Verdaguer T. Las Hualtatas

5951 Vitacura, Santiago de Chile. Fono: (56-2) 3704600.

Fax: (56-2) 371 8934. E mail: oftalandes@entelchile.net 
$\mathrm{L}$ a retinopatía diabética (RD) es una complicación vascular común de la diabetes mellitus tipos 1 y $2^{1}$. Se estima que es la causa más frecuente de nuevos casos de ceguera en adultos ${ }^{2}$. Hay un aumento de la prevalencia de RD a mayor tiempo de duración de la diabetes ${ }^{3}$. Algunos estudios reportan que la $\mathrm{RD}$ está presente en la mayoría de los pacientes con diabetes tipo 1 (DM1) con más de 15 años de duración ${ }^{4,5}$ y virtualmente en todas las personas con diabetes de más de 20 años ${ }^{5,6}$. Otros factores de riesgo asociados con RD son el mal control glicémico ${ }^{3,7}$, mayor edad ${ }^{8}$, severidad de la retinopatía de base y mal control diabético.

El propósito de este estudio es describir la historia natural de la RD en una población chilena con DM1, seguida por un largo periodo por el mismo oftalmólogo y analizar factores asociados con la progresión a enfermedad proliferativa.

\section{MATERIAL Y MÉTODO}

Pacientes con DM1 controlados por el mismo oftalmólogo (J.V.T) entre 1971 y 2008 fueron revisados retrospectivamente. Seguimiento promedio 18,15 años (3-36) con tiempo de diabetes a primera visita oftalmológica de 14,7 años (0-46). Todos evaluados y tratados por diabetólogos. El análisis incluyó a 39 pacientes. El examen clínico y la recolección de los datos fueron obtenidos de registros médicos hasta agosto de 2008. Todos los controles fueron hechos por el mismo retinólogo, usando procedimientos y equipos acordes a los métodos internacionalmente aceptados. El examen incluyó historia médica y examen oftalmológico completo. Se incluyó año de comienzo de diabetes, calidad de control glicémico, nivel de hemoglobina glicosilada A1c (HbA1c), parientes de primer grado con DM1, nefropatía diabética, diálisis, hipertensión arterial, dislipidemia, embarazo, tipo de tratamiento con insulina, historia de hipoglicemia, pie diabético y uso de fármacos antihipertensivos.

La severidad de la RD fue evaluada con la clasificación International Clinical Diabetic Retinopathy Severity Scale?. Pacientes con neovascularización, hemorragia vítrea o prerretinal fueron asignados al grupo de retinopatía diabética proliferante (RDP). Fueron hechas fotografías del fon- do de ojo en todos los pacientes con RD o con drusas duras. La angiografía con fluoresceína fue realizada según necesidad. La agudeza visual mejor corregida (AV) fue registrada en cada control. Proyección de luz y visión cero no fueron consideradas como AV, y fueron excluidas del análisis estadístico ${ }^{10}$.

Un cuestionario redactado por un diabetólogo fue enviado a pacientes y a sus diabetólogos tratantes. La información solicitada incluía año de diagnóstico, tratamiento inicial, tratamiento actual, control metabólico actual, valores de HbA1c desde el diagnóstico, historia de hipoglicemia, medicamentos anti hipertensivos y diagnóstico clínico de pie diabético y nefropatía. Un valor de HbA1c sobre $7 \%$ fue considerado anormal para propósitos estadísticos. Microalbuminura fue definida con niveles entre 30 y $300 \mathrm{mg} /$ día, y nefropatía fue definida por el tratante, de acuerdo a la presencia de hipertensión arterial, albuminuria $>300 \mathrm{mg} /$ día o proteinuria $>0,5 \mathrm{~g} /$ día. Se catalogaron pacientes como control subóptimo o irregular según criterio de diabetólogo tratante o cuando presentaron al menos un valor de HbA1c sobre 7\%.

Se realizó un estudio de casos y controles comparando RD no proliferante (RDNP) con RDP. Se usó el certificado de defunción del Registro Civil de Chile para consignar causa de muerte al seguimiento.

Estadística. Los pacientes con RDP y RDNP fueron comparados con test t de Student y con la prueba de chi-cuadrado. Se calculó razón de probabilidad (OR) con sus respectivos intervalos de confianza al $95 \%$. Valores con un $\mathrm{p}<0,05$ fueron considerados estadísticamente significativos. Para el análisis de los datos se usó el programa SPSS 16.0.

\section{Resultados}

Caracterización de pacientes. Treinta y nueve pacientes fueron incluidos en el análisis. Veinte mujeres (51,3\%). La media de edad a 2008 fue 46,51 años (2670). Media de la edad de inicio de la diabetes fue de 13,51 años (1-31). Media de años con diabetes fue de 32,92 años (17-56). Veintiún (53,8\%) pacientes tenían hipertensión arterial y diez (25,6\%) dislipidemia. Media de años de diabetes para desarrollar hipertensión arterial fue 23,47 años (8-44). Doce pacientes 


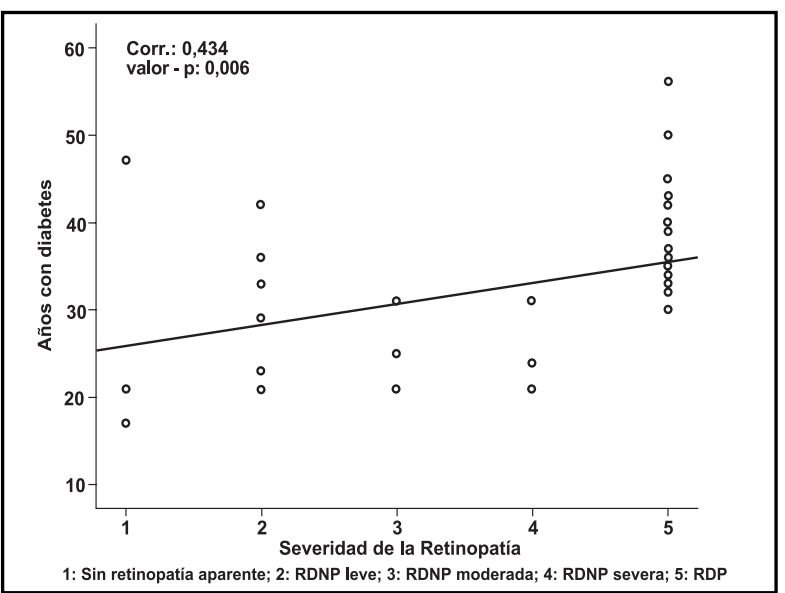

Figura 1. Correlación entre años con diabetes y severidad de la retinopatía.

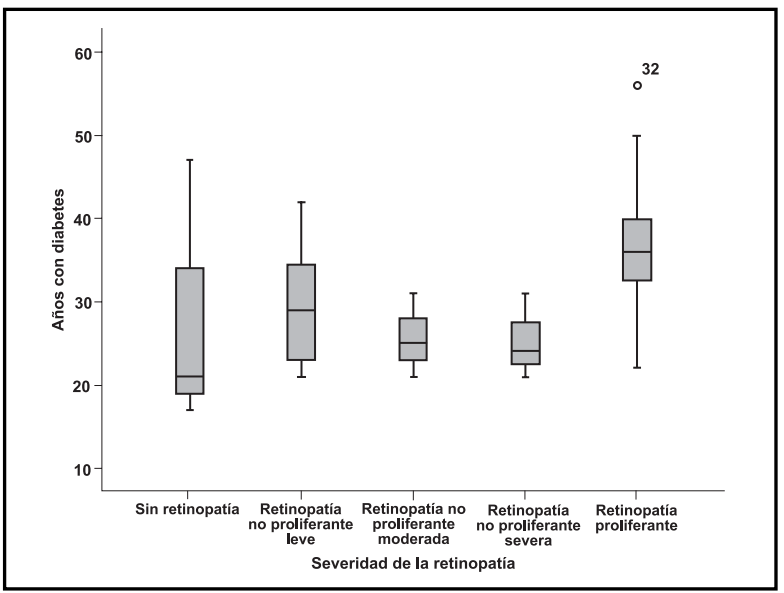

Figura 2. Box-plot. Asociación entre años con diabetes y severidad de la retinopatía. Cada bigote muestra el tiempo mínimo y máximo hasta el diagnóstico de la retinopatía. La línea de la caja muestra la mediana (tiempo hasta que 50\% de los pacientes desarrolló la retinopatía). La amplitud de cada caja y sus bigotes, muestra la variabilidad en el tiempo de diagnóstico de la retinopatía.
$(30,8 \%)$ presentaron microalbuminuria. Cinco $(12,8 \%)$ tenían parientes de primer grado con DM1. Diez desarrollaron cataratas $(25,6 \%)$ y ocho (20,5\%) de ellos requirieron cirugía, con media de años con diabetes para la cirugía de cataratas de 28,13 años (12-45). Tres (7,7\%) pacientes presentaron rubeosis. Nueve $(23,1 \%)$ tuvieron edema macular clínicamente significativo, de los cuales cinco fueron bilaterales y ocho requirieron fotocoagulación focal por esta causa. Un paciente desarrolló glaucoma neovascular.

Retinopatía diabética. De 39 pacientes, 36 (92,3\%) desarrollaron cualquier nivel de RD desde el comienzo de la diabetes hasta el término del seguimiento (Tabla 1). Veintitrés (59\%) alcanzaron RDP. Al ver la asociación entre severidad de la RD y tiempo con diabetes, se obtuvo que la correlación de Spearman era igual a $0,434(\mathrm{p}=0,006)$ (Figura 1). Misma asociación se grafica en Box-Plot (Figura 2). La prevalencia de RDNP y RDP según el tiempo de evolución se muestra en el análisis actuarial (Figuras 3 y 4). Con 5 años de diabetes sólo 1 paciente $(2,7 \%)$ presentaba RD, con 10 años 7 $(17,9 \%)$ tenía RD, con 12 años 11 (28,2\%), con 15 años 19 (48,7\%). Con 20 años de diabetes, $30(76,9 \%)$ presentaba RD, de los cuales 11 (28,2\%) tenían una RDP. No hubo diferencia por sexo para desarrollar RD ( $\mathrm{p}=0,579)$ o RDP ( $\mathrm{p}=0,433)$. Media de edad al momento del diagnóstico de RDP 33,4 años (18-55), y media de duración de la diabetes para desarrollar RDP 20,6 años (10-34). Quince (38,5\%) pacientes presentaron hemorragia vítrea, tres $(7,7 \%)$ desprendimiento de retina traccional y 8 (20,5\%) desarrollaron neovascularización del nervio óptico. Un paciente presentó papilopatía diabética bilateral (Figura 5).

Tabla 1. Severidad de la retinopatía diabética y duración de la diabetes

\begin{tabular}{|lcc|}
\hline Nivel de severidad & $\mathbf{n}$ & $\begin{array}{c}\text { Promedio de la duración de la diabetes en años } \\
\text { (desviación estándar) }\end{array}$ \\
\hline Sin retinopatía aparente & 3 & $28,33(16,3)$ \\
Retinopatía diabética no proliferante leve & 7 & $29,57(7,8)$ \\
Retinopatía diabética no proliferante moderada & 3 & $25,67(5,0)$ \\
Retinopatía diabética no proliferante severa & 3 & $25,33(5,1)$ \\
Retinopatía diabética proliferante & 23 & $36,48(8,1)$ \\
\hline
\end{tabular}


Drusas duras. Se observaron drusas duras en $25(64,1 \%)$ pacientes. En doce casos fueron detectadas y fotografiadas (Figura 6) antes de cualquier manifestación de retinopatía diabética. La media de años con diabetes para la detección de las drusas duras fue de 14,48 (rango: 1-38).

Fotocoagulación panretinal y vitrectomía. Veintitrés pacientes requirieron fotocoagulación panretinal $(58,9 \%)$, obteniéndose estabilización en 20 pacientes, con media de seguimiento desde la fotocoagulación de 13,74 años (4-32). Siete pacientes requirieron vitrectomía.

Agudeza visual final. La AV final convertida a escala LogMAR fue de 0,17 (0-1,3). Entre 3 ojos con visión cero, uno evolucionó a phthisis bulbi post vitrectomía y dos ojos tenían un desprendimiento de retina traccional considerado inoperable.

Embarazo. El 55\% de las mujeres (11 pacientes) se embarazaron al menos en una ocasión. Una paciente sin RD no progresó a ningún nivel de retinopatía. Seis pacientes con retinopatía leve se mantuvieron estables. En cuatro pacientes con RD hubo progresión: un caso de leve a moderado, un caso de moderado a severo y dos casos de leve a PDR. Las pacientes que progresaron a PDR requirieron fotocoagulación agresiva que fue seguida hasta la regresión de la neovascularización.

Trasplante de órganos. Cuatro pacientes estaban en diálisis al momento de cortar este estudio en el año 2008. Un paciente recibió un trasplante de riñón y dos de ellos recibieron un trasplante combinado de páncreas y riñón. Ambos hombres, media de edad 51,5 años, media de años con diabetes 22,5; ambos con RDP al momento del trasplante. En uno la retinopatía progresó a RDP de alto riesgo a dos años del trasplante, a pesar del perfecto control metabólico sin necesidad de medicamentos (Figura 7 a-c).

Control metabólico. La encuesta fue contestada por $24(61,5 \%)$ pacientes y $21(53,8 \%)$ diabetólogos. Combinando ambas encuestas, sólo en 5

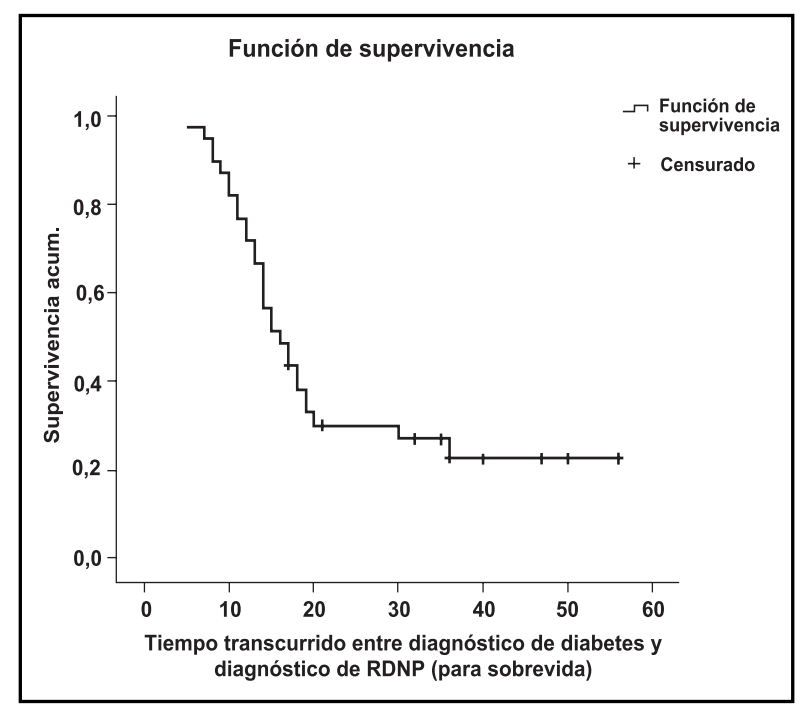

Figura 3. Análisis actuarial. Prevalencia de RDNP según el tiempo de evolución.

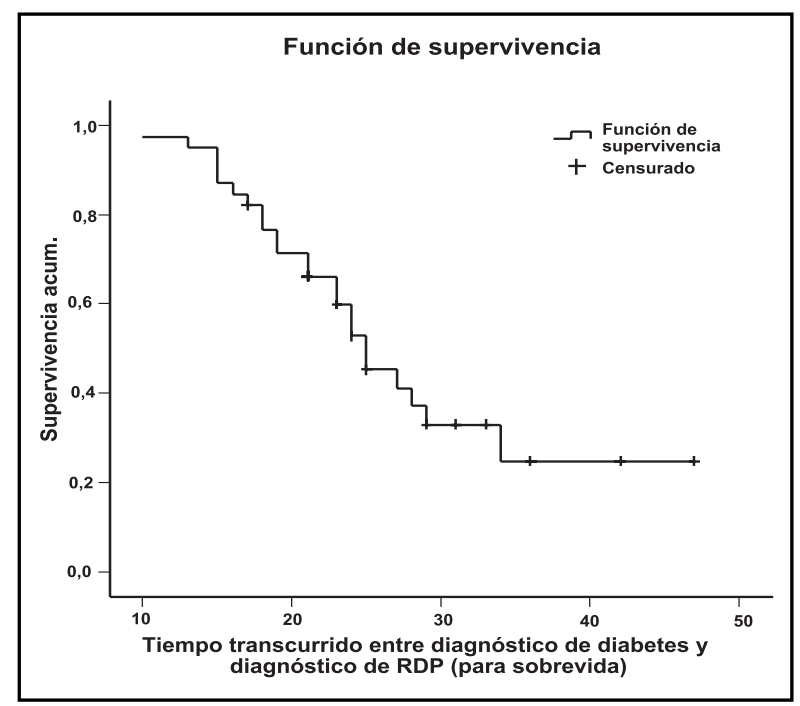

Figura 4. Análisis actuarial. Prevalencia de RDP según el tiempo de evolución.

$(12,8 \%)$ pacientes no se disponía de encuesta contestada. En 20/34 encuestas (58,8\%) el control metabólico fue considerado subóptimo o irregular. En veinte encuestas se declararon episodios ocasionales de hipoglicemia y cinco consignaron episodios frecuentes. La peor HbA1c fue consignada en 37 pacientes. En nueve pacientes el peor 


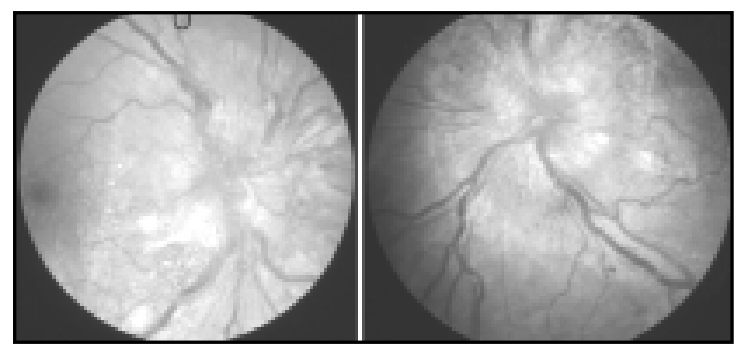

Figura 5. Papilopatía diabética. Fotografía de fondo de (a) ojo derecho y (b) ojo izquierdo. Se observa edema de disco con capilares superficiales dilatados $\mathrm{y}$ tortuosos en una mujer de 17 años.

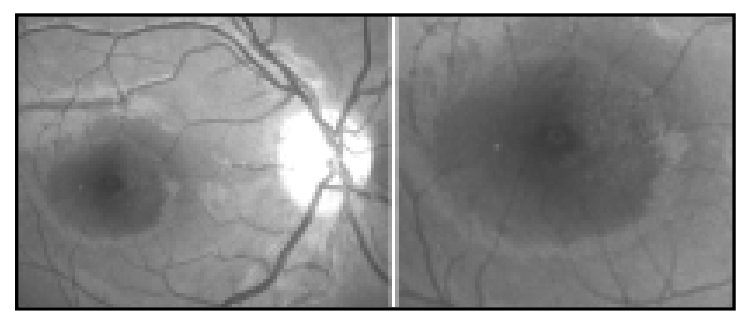

Figura 6. Drusa dura. Fotografía de fondo de ojo de un ojo derecho. (a) Temporal a la fóvea, se observa una drusa dura (flecha). (b) Magnificación de la lesión.

nivel era bajo 7\%. Comparando la presencia de RDP en este grupo $(n=2)$ y en el grupo con el peor nivel de HbA1c sobre 7\% ( $n=19)$, encontramos una diferencia significativa ( $p=0,016) .16$ (41\%) pacientes estaban siendo tratados con inhibidores de la enzima convertidora de angiotensina. Pie diabético fue reportado en $3(7,7 \%)$ pacientes.
Comparación entre pacientes con y sin retinopatía proliferante. Hubo una diferencia significativa en el grupo RDP vs el RDNP para los valores de HbA1c desde el diagnóstico ( $\mathrm{p}=0,011$; $\mathrm{OR}=4,8$; IC $=1,1-$ 20,4 ) y nefropatía ( $\mathrm{p}=0,003$; OR $=10,9$; IC $=1,99-$ 59,72). En grupo RDP hubo significativamente más años con diabetes ( $\mathrm{p}=0,003)$, con media 36,48 \pm 8,15 años de enfermedad, comparado con media $27,81 \pm 8,39$ años de diabetes en grupo RDNP. Los factores asociados a enfermedad proliferante se resumen en la Tabla 2 . También encontramos una mayor frecuencia de drusas duras en el grupo RDNP ( $\mathrm{p}=0,011 ; \mathrm{OR}=0,13 ; \mathrm{IC}=0,02-0,71)$. No hubo diferencias por sexo ( $\mathrm{p}=0,433)$, edad de inicio de diabetes ( $\mathrm{p}=0,546)$, años de seguimiento $(\mathrm{p}=0,173)$, parientes con diabetes tipo $1(\mathrm{p}=0,96)$, cataratas ( $\mathrm{p}=0,117)$, edema macular $(\mathrm{p}=0,191)$, control metabólico presente $(\mathrm{p}=0,563)$, dislipidemia ( $p=0,133)$ y pie diabético $(p=0,727)$. Se observó una tendencia en grupo RDP a significancia estadística para la edad ( $\mathrm{p}=0,061)$, hipertensión arterial ( $\mathrm{p}=0,088)$ y uso de inhibidores de enzima convertidora de angiotensina ( $\mathrm{p}=0,05$; OR $=3,3$; IC $=0,81-13,22)$. Todos los pacientes en diálisis, rubeosis y trasplantes estaban en el grupo de RDP.

Un hombre de 67 años (grupo PDR), después de 50 años con diabetes, falleció de cáncer de colon diseminado.

Tabla 2. Factores asociados a progresión a enfermedad proliferante

Mal control glicémico

Duración de diabetes

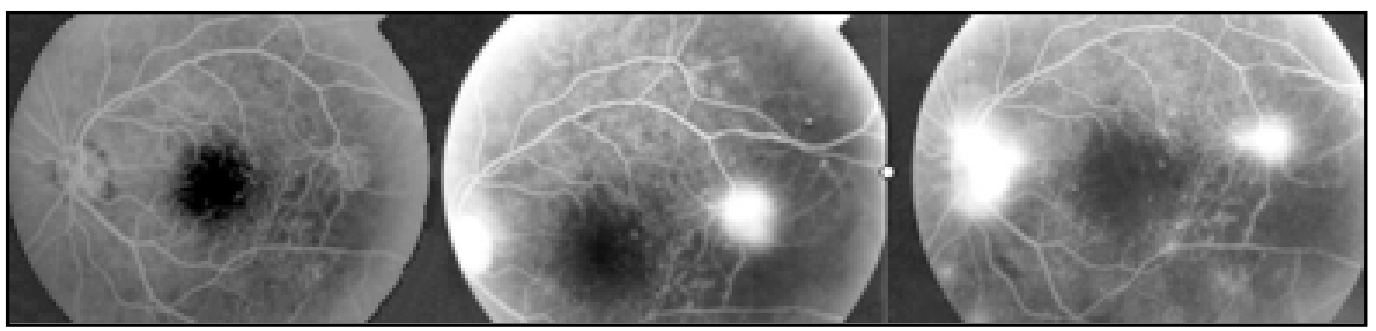

Figura 7. Progresión a retinopatía diabética (RD) de alto riesgo dos años después de un exitoso trasplante combinado de riñón-páncreas. Angiografía con fluoresceína de un hombre de 53 años con un trasplante combinado. (3a) Pequeño penacho neovascular temporal a la mácula sin filtración significativa (angiografía retinal preoperatoria). (3b) Un año después de la cirugía, crecimiento del penacho neovascular, con filtración de fluoresceína. (3c) Dos años después, una RD de alto riesgo se desarrolló, con neovascularización del nervio óptico. 
DisCUSIÓN

Este grupo de 39 pacientes privados con DM1 fueron seguidos por el mismo oftalmólogo por un promedio de 18,15 años. Todos los pacientes pertenecían a la consulta privada de uno de nosotros (J.V.T.); por lo tanto, esta cohorte no es representativa de la población general de DM1 en Chile o América Latina, ya que todos ellos tenían acceso a servicios médicos y tratamientos completos, con niveles educacionales promedio más alto. Historia médica completa, examen ocular y documentación correspondiente estaba disponible en todos los pacientes. Además, los cuestionarios enviados a los pacientes y a sus diabetólogos fueron respondidos en la mayoría $(87,2 \%)$.

Las drusas duras son depósitos globulares de material extracelular hialinizado depositados entre la membrana basal del epitelio pigmentario retinal y la membrana de Bruch. Se les detecta al examen oftalmoscópico como nódulos subretinales puntiformes de color amarillo pálido, redondeados y de bordes netos. En el paciente diabético, deben diferenciarse de los "exudados céreos o duros", que son depósitos de lípidos intrarretinales, de características oftalmoscópicas y significación muy distintas a las drusas duras. En $64,1 \%$ de los pacientes fueron encontradas drusas duras en pequeños números (una a cuatro). En doce fueron detectadas antes del desarrollo de cualquier grado de RD, precediendo el desarrollo de microaneurismas retinales en un promedio de 5,4 años. La duración promedio de la diabetes al tiempo de la detección de las drusas duras fue de 14,48 años. La detección de drusas duras antes del desarrollo de microaneurismas puede indicar un daño temprano del epitelio pigmentario retiniano (EPR) relacionado a la diabetes. Vinores et al reportaron daño ultraestructural progresivo del EPR en ratas BB espontáneamente diabéticas ${ }^{11}$. Mac Gregor et al demostraron una función alterada del EPR en ratas con diabetes inducida por alloxano ${ }^{12}$. Otros autores también han demostrado daño del EPR en animales con diabetes experimental ${ }^{13,14}$.

Es bien sabido y está bien documentado en estudios grandes controlados, que el óptimo control glicémico puede sustancialmente reducir el riesgo de progresión de la $\mathrm{RD}^{7}$. En reportes recientes se han informado prevalencias de RD menores de las esperadas ${ }^{15-20}$, lo que es consis- tente con nuestros hallazgos. En nuestro estudio, a 10 años de diabetes, la prevalencia de RD fue de $17,9 \%$ comparado con $39 \%$ de Le Claire et al ${ }^{16}$. El estudio poblacional Wisconsin Epidemiologic Study of Diabetic Retinopathy, que reclutó pacientes con diabetes entre 1979-1980, reportó una prevalencia de 74\% a los 9-10 años de DM1.

Veintitrés pacientes desarrollaron RD proliferante durante el seguimiento. El análisis estadístico mostró que el riesgo de desarrollar enfermedad proliferativa aumentaba significativamente con la duración. Como era de esperar, la calidad del control glicémico se correlacionó con la severidad de la retinopatía. Hubo una diferencia significativa para los valores alterados de HbA1c desde el diagnóstico. Es importante entonces enfatizar que nuestros resultados son concordantes con lo reportado en la literatura, respecto a los factores de riesgo de progresión a enfermedad proliferante. Respecto a la asociación de RDP con hipertensión arterial, hubo sólo una tendencia, no significativa. Esto puede deberse al pequeño número de pacientes en este estudio.

El análisis entre nefropatía y RD diabética mostró que la nefropatía era más frecuente en el grupo con RDP que en el RDNP. Está bien establecido que la presencia de microalbuminuria $\mathrm{O}$ nefropatía establecida se correlacionan bien con las formas severas de retinopatía ${ }^{21-24}$. Sin embargo, no todos los pacientes con RDP mostraron evidencia de microalbuminuria; de hecho, sólo 13 de 23 pacientes con RDP tuvieron nefropatía diabética demostrable (56\%), a pesar de la larga duración de la diabetes en este grupo (36,5 $\pm 8,2$ años). Esto es consistente con otros estudios; Lövestam-Adrian et $\mathrm{al}^{22}$ siguieron 24 pacientes con RDP por 10 años; sólo 2 desarrollaron microalbuminuria. En ese estudio se concluyó, que al menos parcialmente, hay mecanismos patogénicos distintos para el desarrollo de nefropatía y retinopatía diabética. Romero et $\mathrm{al}^{21}$ hicieron un estudio con 15 años de seguimiento a una cohorte de 112 pacientes con DM1 sin RD o nefropatía al momento de reclutarlos. En los 62 pacientes que desarrollaron retinopatía, sólo 53,2\% de ellos tenía microalbuminuria; en los pacientes con RDP 78\% desarrolló microalbuminuria. En síntesis, aunque hay correlación entre las formas severas de RD y nefropatía, todavía hay un grupo de pacientes con RDP severa que no desarrollan nefropatía. 
Un paciente en este grupo presentó papilopatía diabética. Se trataba de una niña adolescente de 17 años, no adherente al tratamiento, con mal control glicémico. En el examen de rutina se encontró edema de disco bilateral, con capilares superficiales dilatados y tortuosos (Figura 5), con RDNP moderada. El edema resolvió espontáneamente, pero desarrolló una $\mathrm{RD}$ muy severa que requirió un tratamiento agresivo. Perdió un ojo, pero el otro tiene buena visión con estabilización de la retinopatía a 20 años del episodio de papilopatía diabética.

Once pacientes tuvieron embarazo durante el seguimiento. Un caso progresó de retinopatía leve a moderada y un caso de moderada a severa. En dos casos la RD progresó a RDP, requiriendo panfotocoagulación completa. En el seguimiento de la retinopatía durante el embarazo se debe tener un monitoreo cuidadoso, así como también sería útil una consejería y un examen de fondo de ojo previo a un embarazo. Se ha recomendado que las pacientes con DM1 completen su familia lo antes posible ${ }^{25}$.

Dos pacientes que recibieron un trasplante simultáneo de páncreas-riñón merecen un comentario. Ambos pacientes lograron independencia de la insulina y euglicemia sostenida. Un hombre de 50 años tenía una RDP activa, que post trasplante y panfotocoagulación en ambos ojos, evolucionó a una retinopatía estable e inactiva. El segundo paciente era un hombre de 53 años que sólo tenía una RDP leve al momento del trasplante, con un pequeño penacho neovascular temporal a la mácula, sin filtración significativa a la angiografía con fluoresceína (Figura 7a). Un año después de la cirugía, el paciente estaba euglicémico e independiente de insulina, pero el examen oftalmos-

\section{REFERENCIAS}

1. Aiello LP, Cahill MT, Wong JS. Systemic consideratio$\mathrm{ns}$ in the management of diabetic retinopathy. Am J Ophthalmol 2001; 132: 760-76.

2. TAYLOR HR, KeEFFE JE. World blindness: a 21st century perspective. Br J Ophthalmol 2001; 85: 261-6.

3. The Eurodiab Prospective Complications Study Group. Risk factors for progression to proliferative diabetic retinopathy in the EURODIAB Prospective Complications Study. Diabetologia 2001; 44: 2203-9. cópico mostró crecimiento del penacho neovascular, ojo izquierdo, con filtración a la angiografía con fluoresceína (Figura 7b); dos años después del exitoso transplante combinado, desarrolló RD de alto riesgo con neovascularización del disco óptico (Figura 7c); en ese momento se realizó una panfotocoagulación. Estudios controlados han descrito estabilización o mejoría de la RD después de un transplante combinado ${ }^{26}$ o sólo transplante de páncreas ${ }^{27}$, con deterioro sustancial en la mayoría de los ojos usados como controles; en contraste, otros estudios no han podido demostrar diferencia en la progresión de la RD entre transplante de riñón-páncreas y diabéticos receptores de páncreas ${ }^{28-30}$. Chow et al ${ }^{31}$ reportaron progresión de RD in $10 \%$ de 98 ojos, en un estudio a largo plazo de trasplante combinado exitoso. Hemos documentado un caso de progresión a RD de alto riesgo después de dos años de independencia de insulina y euglicemia. No hay explicación satisfactoria para esta progresión. El paciente tenía algunas áreas de no perfusión capilar al momento del trasplante; podría postularse que el cierre capilar no regresa, incluso si la euglicemia sostenida es lograda; la liberación de factores vasoactivos desde esta retina isquémica podría promover el crecimiento de tejido neovascular; esto por supuesto, es sólo especulación.

Este trabajo tiene limitaciones obvias, como todos los estudios retrospectivos. Variables como hipertensión arterial, control metabólico o dislipidemia fueron definidas por el diabetólogo tratante. También, el número de pacientes es pequeño; sin embargo, los pacientes fueron estudiados, documentados y seguidos por el mismo retinólogo, por un periodo de 36 años.

4. Klein R, Klein BE. Diabetic eye disease. Lancet 1997; 350: 197-204.

5. Klein R, Klein BE, Moss SE, Davis MD, Demets DL. The Wisconsin Epidemiologic Study of Diabetic Retinopathy II. Prevalence and risk of diabetic retinopathy when age at diagnosis is less than 30 years. Arch Ophthalmol 1984; 102: 520-6.

6. Goldstein DE, Bunder KJ, Ide CH, Wilson RJ, Wiedmeyer HM, LITTLE RR ET AL. Glycemic control and development of retinopathy in youth-onset insulin-dependent diabetes mellitus. Results of a 12-year longitudinal study. Ophthalmology 1993; 100: 1125-31. 
7. The Diabetes Control and Complications Trial Research Group: The effect of intensive treatment of diabetes on the development and progression of longterm complications in insulin-dependent diabetes mellitus. N Engl J Med 1993; 329: 977-86.

8. Olsen BS, Johannesen J, Sjolie AK, Borch-Johnsen K, Hougarrdss P, Thorsteinsson B et al. Metabolic control and prevalence of microvascular complications in young Danish patients with type 1 diabetes mellitus. Diabet Med 1999; 16: 79-85.

9. Wilkinson CP, Ferris F, Klein R, Lee P, Agardh CD, Davis M ET AL. Proposed International Clinical Diabetic Retinopathy and Diabetic Macular Edema Disease Severity Scales. Ophthalmology 2003; 110: 1677-82.

10. Holladay JT. Visual Acuity Measurement. J Cataract Refract Surg 2004; 30: 287-90.

11. May EE, Blaydes SH. Progressive ultrastructural damage and thickening of the basement membrane of the retinal pigment epithelium in spontaneously diabetic BB rats. Exp Eye Res 1988; 46: 545-58.

12. MacGregor LC, Matschinsky FM. Experimental diabetes mellitus impairs the function of the retinal pigmented epithelium. Metabolism 1986; 35 (4 Suppl 1): 28-34.

13. Aizu Y, Oyanagi K, Hu J, Nakagawa H. Degeneration of retinal neuronal processes and pigment epithelium in the early stage of the streptozotocin-diabetic rats. J Biol Chem 1986; 261: 4046-51.

14. Kirber WM, Nichols CW, Grimes PA, Winegrad AI, Laties AM. A permeability defect of the retinal pigment epithelium. Occurrence in early streptozocin diabetes. Arch Ophthalmol 1980; 98: 725-8.

15. Henricsson M, Nystr L, Blohmé G, Östam J, Kullberg C, Svensson M ET AL. The Incidence of Retinopathy 10 Years After Diagnosis in Young Adult People With Diabetes. Diabetes Care 2003; 26: 349-54.

16. Lecaire T, Palta M, Zhang H, Allen C, Klein R, D'Alessio D. Lower-than-Expected Prevalence and Severity of Retinopathy in an Incident Cohort followed during the First 4-14 Years of Type 1 Diabetes. Am J Epidemiol 2006; 164: 143-50.

17. Skrivarhaug T, Fosmark DS, Stene lC, Bangstad HJ, SANDviK L, Hanssen KF, Joner G. Low cumulative incidence of proliferative retinopathy in childhoodonset type 1 diabetes: a 24-year follow-up study. Diabetologia 2006; 49: 2281-90.

18. Hovind P, Tarnow L, Rossing K, Rossing P, Eising S, Larsen N, Binder C, Parving HH. Decreasing incidence of severe diabetic microangiopathy in type 1 diabetes. Diabetes Care 2003; 26: 1258-64.

19. Nordwall M, Bojestig M, Arnqvist HJ, Ludvigsson J, Linköping Diabetes Complications Study. Declining incidence of severe retinopathy and persisting decrease of nephropathy in an unselected population of type 1 diabetes - The Linkøping Diabetes Complications Study. Diabetologia 2004; 47: 1266-72.

20. Knudsen LL, Lervang H-H, Lundbye-Christensen S, Gorst-Rasmussen A. The North Jutland County Diabetic Retinopathy Study: population characteristics. Br J Ophthalmol 2006; 90; 1404-9.

21. Romero P, Salvat M, Fernández J, Baget M, Martínez I. Renal and retinal microangiopathy after 15 years of follow-up study in a sample of Type 1 diabetes mellitus patients. J Diabetes Complications 2007; 21: 93- 100.

22. Lövestam-Adrian M, Agardh E, Agardh C. The incidence of nephropathy in type I diabetic patients with proliferative retinopathy, a 10 year follow-up study. Diabetes Res Clin Pract 1998; 39: 11-7.

23. Klein R, Moss S, Kuein B. Is gross proteinuria a risk factor for the incidence of proliferative diabetic retinopathy? Ophthalmology 1993; 100: 1140-6.

24. Kofoed-Enevoldsen A, Jensen T, Borch-Johnsen K, Deckert $T$. Incidence of retinopathy in type I (insulin-dependent) diabetes, association with clinical nephropathy. J Diab Complications 1987; 1: 96-9.

25. Rahman W, Rahman F, Yassin S, Al-Suleiman S, Rahman $\mathrm{J}$. Progression of retinopathy during pregnancy in type 1 diabetes mellitus. Clinical and Experimental Ophthalmology 2007; 35: 231-6.

26. Di Landro D, Koenigsrainer L, Oefner D, Aichberger C, Romagnoli GF, Margreiter R. Experience with 100 combined pancreatic renal transplantation in a single center. Nephron 1996; 72: 547-51.

27. Scheider A, Meyer-Schwickerath V, Nusser J, Land W, LANDGRAF R. Diabetic retinopathy and pancreas transplantation: a 3-year follow-up. Diabetologia 1991; 34 (Suppl. 1): S95-99.

28. Caldara R, Bandello F, Vigano C, Secchi A, Castoldi R, CALDI M ET AL. Influence of successful pancreaticorenal transplantation on diabetic retinopathy. Transpl Proc 1994; 26: 490.

29. Petersen MR, Vine AK. Progression of diabetic retinopathy after pancreas transplantation. Ophthalmology 1990; 97: 496-500.

30. Wang Q, Klein R, Moss Se, Kuein Be, Hoyer C, Burke K ET AL. The influence of combined kidney-pancreas transplantation on the progression of diabetic retinopathy. Ophthalmology 1994; 101: 1071-6.

31. Chow VCC, Pai RP, Chapman Jr, O'Connell PJ, Allen RDM, Mitchell P et al. Diabetic retinopathy after combined kidney-pancreas transplantation. Clin Transplantation 1999; 13: 356-62. 